\title{
Optimal Homotopy Asymptotic Method-Least Square for Solving Nonlinear Fractional-Order Gradient-Based Dynamic System from an Optimization Problem
}

\author{
Oluwaseun Olumide Okundalaye, Wan Ainun Mior Othman $\mathbb{D}$, \\ and Nallasamy Kumaresan
}

Institute of Mathematical Sciences, University of Malaya, 50603 Kuala Lumpur, Malaysia

Correspondence should be addressed to Wan Ainun Mior Othman; wanainun@um.edu.my

Received 4 March 2020; Revised 6 June 2020; Accepted 10 June 2020; Published 26 July 2020

Academic Editor: Remi Léandre

Copyright (C) 2020 Oluwaseun Olumide Okundalaye et al. This is an open access article distributed under the Creative Commons Attribution License, which permits unrestricted use, distribution, and reproduction in any medium, provided the original work is properly cited.

\begin{abstract}
In this paper, we consider an approximate analytical method of optimal homotopy asymptotic method-least square (OHAM-LS) to obtain a solution of nonlinear fractional-order gradient-based dynamic system (FOGBDS) generated from nonlinear programming (NLP) optimization problems. The problem is formulated in a class of nonlinear fractional differential equations, (FDEs) and the solutions of the equations, modelled with a conformable fractional derivative (CFD) of the steepest descent approach, are considered to find the minimizing point of the problem. The formulation extends the integer solution of optimization problems to an arbitrary-order solution. We exhibit that OHAM-LS enables us to determine the convergence domain of the series solution obtained by initiating convergence-control parameter $C_{j}^{\prime}$ s. Three illustrative examples were included to show the effectiveness and importance of the proposed techniques.
\end{abstract}

\section{Introduction}

Consider a nonlinear programming-constrained optimization problems (NLPCOPs) of the form

$\min _{x \in \Re^{n}} f(x)$ subject to $g_{k}(x) \leq 0$ and $h_{k}(x)=0 \forall k \in I=\{1,2 . . m\}$,

where $f: \mathfrak{R}^{n} \longrightarrow \mathfrak{R}, h_{k}: \mathfrak{R}^{n} \longrightarrow \mathfrak{R}$, and $g_{k}: \mathfrak{R}^{n} \longrightarrow \mathfrak{R}, k$, are $C^{2}$ functions. Let $X_{0}=\left\{x \in \mathfrak{R}^{n} \mid h_{k}=0, g_{k} \leq 0, i \in I\right\}$ be the feasible set of Equation (1), and we assume that $X_{0}$ is not empty. The general idea of obtaining an approximate analytical solution to Equation (1) is to transform to an unconstrained nonlinear programming problem by any suitable technique such as augmented Lagrange method, barrier method, and penalty method $[1,2]$; it can then be solved by any unconstrained optimization numerical method like the steepest descent method, conjugate gradient method, and Newton method. In optimization, the penalty method is the most efficient method to transform a constrained optimization problem into an unconstrained optimization problem [3-5]. An efficient penalty function for equality and inequality problem Equation (1) is given below

$$
\begin{aligned}
& P_{\text {penalty }}\left(h_{k}(x)\right)=\mu \frac{1}{\sigma} \sum_{i=1}^{p}\left(h_{k}(x)\right)^{\sigma}, \\
& P_{\text {penalty }}\left(g_{k}(x)\right)=\mu \sum_{i=1}^{p}\left(\max \left\{0, g_{k}(x)\right\}\right)^{\sigma},
\end{aligned}
$$

where $\sigma=2$. It can be seen that under some conditions, the solutions to Equation (1) are solutions of the unconstrained below [6], 


$$
\begin{aligned}
& \min F(x, \mu)=f(x)+\mu\left(\frac{1}{\sigma} \sum_{i}^{p}\left(h_{k}(x)\right)^{\sigma}+\sum_{i=1}^{p}\left(\max \left\{0, g_{k}(x)\right\}\right)^{\sigma}\right), \\
& \text { subject to } \quad x \in \mathfrak{R}^{n},
\end{aligned}
$$

where $\mu>0$ is an auxiliary penalty variable. The corollary connecting the minimizer of the constraint problem in Equation (1) and unconstrained problem in Equation (4) is seen in [7]. The gradient descent method as a standard optimization algorithm has been widely applied in many engineering applications, such as optimization machine learning and image [8-10]. Through diverse research and studies, it is established that the gradient method is one of the most reliable and efficient ways to find the optimal solution of optimization problems [11]. Nowadays, one of the critical points of the gradient method is how to improve the performance further. As an important area of mathematics, fractional calculus is believed to be an excellent tool to enhance the old gradient descent method, mainly because of its special long memory characteristics and nonlocality [12-14]. In the past decade, several methods have been considered to solve unconstrained nonlinear optimization in the form of ordinary differential equation (ODE) dynamic system of which the gradient-based method is one of the approaches. The technique transforms the nonlinear optimization problem to an ODE dynamic system with some optimality conditions, to obtain optimal solutions to the optimization problem. The gradient-based method was first proposed by [15], was developed by $[16,17]$, and was later extended to solve differential nonlinear programming problems [18]. However, the studies of nonlinear fractional-order gradient-based dynamic systems are still in the infant stage and are considered further in this paper.

Arbitrary-order ODEs, which are the generalizations of integer-order ODEs, are mostly used to model problems in applied sciences. Several numerical methods had been used to solve linear and nonlinear problems of FDEs, such as the Adomian decomposition method (ADM) [19], variational iteration method (VIM) [20], homotopy perturbation method for solving fractional Zakharov-Kuznetsov equation [21], a numerical method for FDEs [22], and multivariate padé approximation (MPA) [23]. The usefulness of an arbitrary-order started receiving tremendous attention of researchers in the field of applied science and engineering in the last two decades where some authors in the area of optimization focused on developing approximate analytical methods for different types of nonlinear constrained optimization problems in the form of IVPs of nonlinear FDE systems including multistage ADM for NLP [24], a fractional dynamics trajectory approach [25], the convergence of HAM and application [26], fractional steepest descent approach [27], studied optimal solution of fractional gradient [28], gradient descent direction with Caputo derivative sense for BP neural networks [29], fractional-order gradient methods [30], and conformable fractional gradient-based system [31]. In 2008, Marinca and Herisanu [32] introduced a numerical method called OHAM to solve a nonlinear problem, later extended by Azimi et al. [33] for strong nonlinear differential equations (NLDEs). This powerful tool called OHAM has not been applied in the area of FOGBDS, which motivates this work.

So, in this paper, we showed that the steady-state solutions $x(t)$ of the proposed system can be approximated analytically to the expected exact optimal solution $x^{*}$ of the nonlinear programming constrained optimization problem by OHAM-LS as $t \longrightarrow \infty$. The significant contribution is summarized as follows:

(1) The reason why OHAM-LS is preferable to be the method used $[25,31]$ to solve FOGBDS

(2) The reason why some existing approximate analytical method cannot guarantee the convergence of the series solution is discussed

(3) From the previous approximation analytical method of solving FOGBDS, accurate optimal values control-convergence parameter had been a little bit difficult to achieve which is easily address with least square optimization techniques

(4) OHAM-LS with guaranteed convergence ability is proposed with conformable fractional derivative sense to solve FOGBDS. The fastest convergence ability of the proposed compared with fourth-order Runge-Kutta is also shown

We arrange the paper as follows: a brief introduction to the fractional calculus and OHAM-LS derivation is given in Section 2. Section 3 is devoted to problem formulation of OHAM-LS with FOGBDS and the key contributions. In Section 4, we solved some NLP constrained optimization problems to show the effectiveness of the proposed method. The results obtained from OHAM-LS are plotted in several figures with numerical method comparisons to confirm the validity and ability of the method to solve the problem. In the last section are the conclusions.

\section{Preliminaries}

2.1. Fractional Calculus. The most common arbitrary-order in literature is the Riemann-Liouville's and the Caputo fractional derivative. The arbitrary-order definitions are generally used for mathematical modelling within many areas, especially when the classical-order derivative operator fails or additional memory effect is required. However, the limitation of these two definitions is that they do not provide some of the features that the classical derivative provides, such as chain rule, quotient rule, product rule, and derivative of 
constant. Recently, Khalil et al. [34] have characterized a new fractional derivative operator, which is an extension of the usual conformable fractional derivative, to overcome these deficiencies. Besides these advantages, the conformable fractional derivative does not show the memory effect, which is inherent for the other classical fractional derivatives.

Definition 1. Let $f:[0, \infty) \longrightarrow \mathfrak{R}$ be a given function. The $\alpha^{\text {th }}$ order CFD of $f$ given by

$$
T^{\alpha}(f)(x)=\lim _{\varepsilon \rightarrow 0} \frac{f\left(x+\varepsilon x^{1-\alpha}\right)-f(x)}{\varepsilon},
$$

$\forall x>0$ and $\alpha \in(0,1]$

This new definition preserves many properties of the classical derivatives refer to $[34,35]$. Some features that we will adopt are as follows:

Theorem 2. Let $0<\alpha \leq 1$ and $(f, g)$ be $\alpha$-differentiable at a point $x>0$; if $f$ is a differentiable function, then $\left(d^{\alpha} f\right) /\left(d x^{\alpha}\right)$ $=x^{1-\alpha}(d f / d x)$.

Definition 3. $I_{a}^{\alpha}(f)(x)=I_{a}^{1}\left(x^{\alpha-1} f\right)=\int_{a}^{x}\left((f(t)) /\left(t^{1-\alpha}\right)\right) d t$, where the integral is the regular Riemann improper integral, and $\alpha \in(0,1]$.

Theorem 4. Let $f$ be any continuous function in the domain of $I^{\alpha}$, then $T^{\alpha} I_{a}^{\alpha}(f)(x)=f(x) \forall x \geq a$.

Theorem 5. Let $f:(a, b) \longrightarrow \Re$ be differentiable and $0<\alpha$ $\leq 1$. Then, for all $x>a$, we have $I_{a}^{\alpha} T^{\alpha}(f)(x)=f(x)-f(a)$.

2.2. The Elementary Concepts of OHAM-LS. We start from the fundamental principle of OHAM as described in [3638]. Consider the IVPs

$$
L_{i}\left(z_{i}(t)\right)+N_{i}\left(z_{i}(t)\right)+g_{i}(t)=0 t \in \varphi i=1,2, \cdots m
$$

with initial conditions

$$
z_{i}(b)=a_{i}
$$

where $L_{i}$ is a linear operator, $N_{i}$ is a nonlinear operator, $t$ is an independent variable, $z_{i}(t)$ is an unknown function, $\varphi$ is the problem domain, and $g_{i}(t)$ is a known function. According to OHAM, one can construct an homotopy map $H_{i}\left(\phi_{i}(t, p): \varphi \times[0,1] \longrightarrow \varphi\right.$ which satisfies

$$
\begin{aligned}
& (1-p)\left[L_{i}\left(\phi_{i}(t, p)\right)+g_{i}(t)\right] \\
& \quad=H_{i}(p)\left[L_{i}\left(\phi_{i}(t, p)\right)+N_{i}\left(\phi_{i}(t, p)\right)+g_{i}(t)\right],
\end{aligned}
$$

where $p \in[0,1]$ is an embedding parameter, $H_{i}(p)$ is a nonzero auxiliary function for $p \neq 0, H(0)=0$, and $\phi_{i}(t, p)$ is an unknown function. Obviously, when $p=0$ and $p=1$, it holds that $\phi_{i}(t, 0)=z_{i, 0}(t)$ and $\phi_{i}(t, 1)=z_{i}(t)$, respectively. Thus, as $p$ varies from 0 to 1 , the solution $\phi_{i}(t, p)$ approaches from $z_{i, 0}(t)$ to $z_{i}(t)$ where $z_{i, 0}(t)$ is the initial guess that satisfies the linear operator which is obtained from Equation (8) for $p=0$ as

$$
L_{i}\left(z_{i, 0}(t)+g_{i}(t)\right)=0 . z_{i, 0}(b)=0 \text {. }
$$

$H_{i}(p)$ is chosen in the form

$$
H_{i}(p)=p C_{1}+p^{2} C_{2}+p^{3} C_{3}+\cdots \quad j=1,2, \cdots n,
$$

where $C_{j}$ would be determined in the last part of this work. We consider Equation (8) in the form

$$
\phi_{i}\left(t, p, C_{j}\right)=z_{i, 0}(t)+\sum_{k \geq 1} z_{i, k}\left(t, C_{j}\right) p^{k} \quad j=1,2, \cdots n .
$$

Now substituting Equation (11) in Equation (8) and equating the coefficient of like power of $p$, we obtain the governing equation of $z_{i, 0}(t)$ in a linear form, given in Equation (9). The first- and second-order problems are given by

$$
\begin{aligned}
& L_{i}\left(z_{i, 1}(t)\right)+g_{i}(t)=C_{1} N_{0}\left(z_{i, 0}(t)\right), \quad z_{i, 1}(b)=0, \\
& L_{i}\left(z_{i, 2}(t)\right)-L_{i}\left(z_{i, 1}(t)\right)=C_{2} N_{i, 0}\left(z_{i, 0}(t)\right)+C_{1}\left[L_{i}\left(z_{i, 1}(t)\right)\right. \\
& \left.+N_{i, 1}\left(z_{i, 1}(t)\right)\right], \quad z_{i, 2}(b)=0 \text {, }
\end{aligned}
$$

and the general governing equations for $z_{i, k}(t)$ are given by

$$
\begin{aligned}
& L_{i}\left(z_{i, k}(t)\right)-L_{i}\left(z_{i, k-1}(t)\right) \\
& =C_{k} N_{i, 0}\left(z_{i, 0}(t)\right)+\sum_{m=1}^{k-1} C_{j, m}\left[L_{i}\left(z_{i, k-m}(t)\right)\right. \\
& \left.\quad+N_{i, k-m}\left(z_{i, k-1}(t)\right)\right], \\
& z_{i, k}(b)=0, \quad k=2,3, \cdots,
\end{aligned}
$$

where $N_{i, m}\left(z_{0}(t), z_{i, 1}(t) \cdots, z_{i, m}(t)\right)$ is the coefficient of $p^{m}$, obtained by expanding $N_{i}\left(\phi_{i}\left(t, p, C_{j}\right)\right)$ in series with respect to the embedding parameter $p$

$$
N_{i}\left(\phi_{i}\left(t, p, C_{j}\right)\right)=N_{i, 0}\left(z_{i, 0}(t)\right)+\sum_{m=1}^{\infty} N_{i, m}\left(z_{i, m}(t)\right) p^{m}
$$

where $\phi_{i}\left(t, p, C_{j}\right)$ is obtained from Equation (11). It should noted that $z_{i, k}$ for $k \geq 0$ is governed by the linear Equations (9), (12), and (14) with linear initial conditions that come from the original problem, which can be easily solved.

It has been shown that the convergence of the series Equation (16) depends upon the $C_{j}$. If it is convergent at $p=1$, we have

$$
z_{i}\left(t, C_{j}\right)=z_{i, 0}(t)+\sum_{k \geq 1} z_{i, k}\left(t, C_{j}\right)
$$

The result of the $m$ th-order approximation is given as

$$
\tilde{z}_{i}\left(t, C_{j}\right)=z_{i, 0}(t)+\sum_{k=1}^{m} z_{i, k}\left(t, C_{j}\right)
$$


Substituting Equation (18) in Equation (6), we get the following expression for the residual

$$
R_{i}\left(t, C_{j}\right)=L_{i}\left(\tilde{z}_{i}\left(t, C_{j}\right)+N_{i}\left(\tilde{z}_{i}\left(t, C_{j}\right)+g_{i}(t), \quad i=1,2, \cdots m,\right.\right.
$$

If $R_{i}\left(t, C_{j}\right)=0$, then $\tilde{z}_{i}\left(t, C_{j}\right)$ is the exact solution. Usually, such a case does not arise for nonlinear problems. Several methods $[39,40]$ can be used to find the optimal values of convergence-control parameters $C_{j}^{\prime} s$ like the method of the least square method, collocation method, Ritz method, and Galerkin's method. By applying the least square method, we have minimized the functional

$$
J_{k}\left(C_{1}, C_{2}, C_{3}, \cdots C_{m}\right)=\int_{a}^{b} R_{k}^{2}\left(t, C_{1}, C_{2}, C_{3}, \cdots C_{m}\right) d t
$$

where the value $a$ and $b$ depends on the given problem.

$$
\psi_{k}=\frac{\partial J_{k}\left(C_{k}\right)}{\partial C_{k}}=0, \quad k=1,2, \cdots m
$$

With these known $C_{j}^{\prime} s$, the approximate solution (of $m$ th-order) is well determined.

The correctness of the method by

(1) Error Norm $L_{2}$.

$$
L_{2}=\left\|Z^{\text {exact }}-Z_{N}\right\| \approx \sqrt{\frac{b-a}{N} \sum_{i=0}^{N}\left|z_{i}^{\text {exact }}-\left(z_{N}\right)_{i}\right|^{2}}
$$

(2) Error Norm $L_{\infty}$.

$$
L_{\infty}=\left\|Z^{\text {exact }}-Z_{N}\right\|_{\infty} \approx \max _{i}\left|Z_{i}^{\text {exact }}-\left(Z_{N}\right)_{i}\right|
$$

The OHAM-LS is based on hybridization of OHAM with the least square method of optimization technique. The OHAM enable us to determine the convergence domain of the series solution, and the least square method allows us to obtain the optimal values of the $C_{k}^{s}$.

Remark 6. OHAM-LS is preferable because VIM, HPM, and HAM are just a case as proved by [41-44].

Remark 7. The existing approximate analytical for FOGBDS cannot guarantee convergence mainly because they possess no criteria for the establishment for convergence of the series solution Equations (20) and (21).

\section{Construction of OHAM-LS with FOGBDS Generated by NLPCOPs}

We begin by considering a NLP constrained in the form

$\min _{x \in \Re^{n}} f(x)$ subject to $g_{k}(x) \leq 0$ and $h_{k}(x)=0 \forall k \in I=\{1,2 . . m\}$,

where $f: \mathfrak{R}^{n} \longrightarrow \Re$ is the objective function, $h_{k}(x): \mathfrak{R}^{n}$ $\longrightarrow \mathfrak{R}$ are equality constraint functions, $g_{k}(x): \mathfrak{R}^{n} \longrightarrow \mathfrak{R}$ are inequality constraint functions, and $C^{2}$ are continuous differentiable functions. One of the main ideas of solving unconstrained NLP is by searching for the next point by choosing proper search direction $d_{k}$ and the stepsize $\alpha_{k}$ as in the Newton direction [45], trust-region algorithm for unconstrained optimization [46]; the descent method [47], conjugate gradient method [48], three-term conjugate gradient method [49], and subspace method for nonlinear optimization [50]; the hybrid method for convex NLP [51]; CCM for optimization problem and application [52]; and descent direction stochastic approximation for optimization problem [53]. But there are studies for other approaches. In this paper, we obtain the minimizing point of the problem by solving a certain initial-value system of FDEs. This kind of FOGBDS was first proposed by Evirgen and Özdemir [24].

Using the penalty function Equation (2) and (3) for Equation (24) with $\rho=2$, the conformable FOGBDS model can be constructed as

$$
T^{\alpha} x(t)=-\nabla_{x} F(x, \mu),
$$

subject to the initial conditions

$$
x_{k}(0)=x_{k 0}, \quad k=1 \cdots, m \text {. }
$$

where $\nabla_{x} F(x, \mu)$ is the gradient vector of Equation (25) with respect to $x_{k} \in \mathfrak{R}^{n}$ and $T^{\alpha}$ is the CFD of $0<\alpha \leq 1$.

Note that a point $x_{e}$ is called an equilibrium point of Equation (25) if it satisfies the RHS of Equation (25). We reformulate fractional dynamic system Equation (25) as

$$
T^{\alpha} x_{k}(t)=g_{k}\left(t, \mu, x_{1}, x_{2} \cdots . x_{n}\right), \quad k=1,2 \cdots, m \text {. }
$$

We used OHAM-LS to obtain the solution of system Equation (27) by constructing the following homotopy

$$
T^{\alpha} x_{k}(t)=p g_{k}\left(t, \mu, x_{1}, x_{2} \cdots . x_{n}\right),
$$

where $k=1,2 \cdots, n$ and $p \in[0,1]$. If $p=0$, Equation (28) becomes

$$
T^{\alpha} x_{k}(t)=0
$$

and when $p=1$, the homotopy Equation (28) becomes

$$
\begin{aligned}
& T^{\alpha} x_{k}(t)=g_{k}\left(t, \mu, x_{1}, x_{2} \cdots . x_{n}\right), \\
& k=1,2 \cdots . m, t \in[0,1], 0<\alpha \leq 1,
\end{aligned}
$$


subject to the initial conditions,

$$
x_{k}(b)=a_{k}, \quad k=1,2 \cdots, m .
$$

The correction functional for the system of conformable fractional nonlinear differential equation Equation (30), according to OHAM-LS, can be constructed as

$$
\begin{aligned}
(1-p)\left[T^{\alpha}\left(\varphi_{k}(t, p)\right)\right]= & H_{k}(p)\left[T^{\alpha} \varphi_{k}(t, q)+N \varphi_{k}(t, q)\right. \\
& +g_{k}\left(t, \mu, \varphi_{1}(t, q), \varphi_{2}(t, q) \cdots \cdots \varphi_{n}(t, q)\right],
\end{aligned}
$$

Thus as $p$ varies from 0 to 1 , the solution $\varphi_{k}(t, p)$ approaches from $x_{k, 0}(t)$ to $x_{k}(t)$ where $x_{k, 0}(t)$ is the initial guess that satisfies the linear operator which is obtained from Equation (32) for $p=0$ as

$$
T^{\alpha}\left(x_{k, 0}(t)\right)=0, \quad x_{k, 0}(b)=0 .
$$

$H_{k}(p)$ is chosen in the form

$$
H_{k}(p)=p C_{1}+p^{2} C_{2}+p^{3} C_{3} \cdots,
$$

where $C_{j}$ can be determined later. We get an approximate solution by expanding $\varphi_{k}\left(t, p, C_{j}\right)$ in Taylor's series with respect to $p$; we have

$$
\varphi_{k}\left(t, p, C_{j}\right)=x_{i, 0}(t)+\sum_{i \geq 1} x_{k, i}\left(t, C_{j}\right) p^{i}, j=1,2, \cdots, n .
$$

Now using Equation (35) in Equation (32) and equating the coefficient of like power of $p$, we obtain the governing equation of $x_{i, 0}(t)$ in a linear form, given in Equation (33). The 1st- and 2nd-order problems are given by

$$
\begin{aligned}
& T^{\alpha}\left(x_{k, 1}(t)\right)+g_{k}(t)= C_{1} N_{0}\left(x_{k, 0}(t)\right), x_{k, 1}(b)=0, \\
& T^{\alpha}\left(x_{k, 2}(t)\right)-T^{\alpha}\left(x_{k, 1}(t)\right) \\
&= C_{2} N_{k, 0}\left(x_{k, 0}(t)\right)+C_{1}\left[T^{\alpha}\left(x_{k, 1}(t)\right)\right. \\
&\left.+N_{k, 1}\left(x_{k, 1}(t)\right)\right], x_{k, 2}(b)=0
\end{aligned}
$$

and the general governing equations for $x_{k, i}(t)$ are given by

$$
\begin{aligned}
& T^{\alpha}\left(x_{k, i}(t)\right)-T^{\alpha}\left(x_{k, i-1}(t)\right) \\
& =C_{i} N_{k, 0}\left(x_{k, 0}(t)\right)+\sum_{m=1}^{i-1} C_{j, m}\left[T^{\alpha}\left(x_{k, i-m}(t)\right)\right. \\
& \left.\quad+N_{k, i-m}\left(x_{k, i-1}(t)\right)\right], \quad x_{k, i}(b)=0, i=2,3, \cdots m,
\end{aligned}
$$

where $N_{k, m}\left(x_{0}(t), x_{k, 1}(t) \cdots, x_{k, m}(t)\right)$ is the coefficient of $p^{m}$, obtained by expanding

$N_{k}\left(\varphi_{k}\left(t, p, C_{j}\right)\right)$ in series with respect to $p$.

$$
N_{k}\left(\varphi_{k}\left(t, p, C_{j}\right)\right)=N_{k, 0}\left(x_{k, 0}(t)\right)+\sum_{m \geq 1} N_{k, m}\left(x_{0}, x_{1}, \cdots x_{m}\right) p^{m} .
$$

It has been shown that the convergence of the series Equation (38) depends upon the $C_{j}$. If it is convergent at $p=1$, one has

$$
x_{k}\left(t, C_{j}\right)=x_{k, 0}(t)+\sum_{i \geq 1} x_{k, i}\left(t, C_{j}\right) .
$$

The solution of Equation (30) is determined approximately in the form,

$$
\tilde{x}_{k}\left(t, C_{j}\right)=x_{k, 0}(t)+\sum_{i=1}^{m} x_{k, i}\left(t, C_{j}\right), \quad j=1,2, \cdots, n .
$$

Substituting Equation (40) in Equation (30), we get the following expression for the residual error

$$
R_{k}\left(t, C_{j}\right)=T^{\alpha}\left(\tilde{x}_{k}\left(t, C_{j}\right)\right)+N\left(\tilde{x}_{k}\left(t, C_{j}\right)\right)+g_{k}\left(\tilde{x}_{k}\left(t, C_{j}\right)\right) .
$$

If $R_{k}\left(t, C_{j}\right)=0$, then $\tilde{x}_{k}\left(t, C_{j}\right)$ is the exact solution. Usually, such a case does not arise for nonlinear problems. Using the least square method as below minimizes the functional

$$
J_{k}\left(C_{1}, C_{2}, C_{3}, \cdots C_{m}\right)=\int_{a}^{b} R_{k}^{2}\left(t, C_{1}, C_{2}, C_{3}, \cdots C_{m}\right) d t,
$$

where the value of $a$ and $b$ depends on the given problem.

$$
\psi_{k}=\frac{\partial J_{k}\left(C_{k}\right)}{\partial C_{k}}=0, \quad k=1,2, \cdots m .
$$

With these known $C_{k}$, the analytical approximate solution (of $m$ th-order) is well determined.

The steps for optimal homotopy asymptotic methodleast square (OHAM-LS) are as follows:

Step 1. We transform the nonlinear constrained optimization problem to the unconstrained optimization problem by a penalty method.

Step 2. We find the gradient of the unconstrained optimization problem, with given initial conditions.

Step 3. We choose the linear and nonlinear operators for OHAM-LS.

Step 4. We construct homotopy for the conformable fractional nonlinear differential equation which includes embedding parameter, auxiliary function, and the unknown function.

Step 5. We substitute the series solution results into the governing equation and equate to zero for an exact solution. Usually, such case a does not arise in nonlinear problems. 
Step 6. We find the optimal values for $C_{j}^{\prime}$ s by using the optimization method called least square method, for good analytical approximate solution.

\subsection{Convergence Analysis of OHAM-LS with FOGBDS}

Theorem 8. As long as the series $\tilde{x}_{k}\left(t, C_{j}\right)=x_{k, 0}(t)+\sum_{i=1}^{m} x_{k, i}$ $\left(t, C_{j}\right), j=1,2, \cdots, n$ converges where $\tilde{x}_{k}\left(t, C_{j}\right)$ is governed by Equation (40) under the definitions Equations (37) and (38), it must be the solution of Equations (25) and (26).

Proof. If we assume $\sum_{m=1}^{\infty} \tilde{x}_{k, m}\left(t, C_{j}\right), k=1,2 \cdots . n$, converges to $\tilde{x}_{k}\left(t, C_{j}\right)$, then

$$
\lim _{m \rightarrow \infty} \tilde{x}_{k, m}\left(t, C_{j}\right)=0 \forall k=1,2 \cdots n .
$$

From Equation (37), we can write

$$
\begin{aligned}
\sum_{i=1}^{\infty} & {\left[C_{i} N_{k, 0}\left(x_{k, 0}(t)\right)+\sum_{m=1}^{i-1} C_{j, m}\left[T^{\alpha}\left(x_{k, i-m}(t)\right)+N_{k, i-m}\left(x_{k, i-1}(t)\right)\right]\right.} \\
= & \sum_{i=1}^{\infty}\left[T^{\alpha}\left(x_{k, i}(t)\right)-T^{\alpha}\left(x_{k, i-1}(t)\right)\right] \\
= & \lim _{n \rightarrow \infty} \sum_{i=1}^{n} T^{\alpha}\left(x_{k, i}(t)\right)-T^{\alpha}\left(x_{k, i-1}(t)\right) \\
= & T^{\alpha} x_{11}(t)+\left(T^{\alpha} x_{22}(t)-T^{\alpha} x_{21}(t)\right)+. . \\
& +\left(T^{\alpha} x_{n n}(t)-T^{\alpha} x_{n(n-1)}(t)\right) \\
= & T^{\alpha}\left[\lim _{n \rightarrow \infty} \sum_{m=1}^{n} x_{n n}(t)\right]=T^{\alpha}\left[\lim _{n \rightarrow \infty} x_{n n}(t)\right]=0, \\
\sum_{i=1}^{\infty} & {\left[C_{i} N_{k, 0}\left(x_{k, 0}(t)\right)+\sum_{m=1}^{i-1} C_{j, m}\left[T^{\alpha}\left(x_{k, i-m}(t)\right)\right.\right.} \\
& \left.+N_{k, i-m}\left(x_{k, i-1}(t)\right)\right]=0 .
\end{aligned}
$$

So, using above gives

$$
\begin{aligned}
= & \sum_{m=1}^{\infty}\left[T^{\alpha} x_{k(m-1)}+N\left(x_{k(m-1)}\right)\right. \\
& +g_{k}\left(t, \mu, x_{1(m-1)}, x_{2(m-1)} \cdots . . x_{n(m-1)}\right] \\
= & \sum_{m=1}^{\infty} T^{\alpha} x_{k(m-1)}+\sum_{m=1}^{\infty} N\left(x_{k(m-1)}\right) \\
& +\sum_{m=1}^{\infty} g_{k}\left(t, \mu, x_{k(m-1)}\right) \\
= & T^{\alpha} \tilde{x}_{k}\left(t, C_{j}\right)+N\left(\tilde{x}_{k}\left(t, C_{j}\right)\right)+g_{k}\left(\tilde{x}_{k}\left(t, C_{j}\right)\right) .
\end{aligned}
$$

From Equation (48), we have

$T^{\alpha} \tilde{x}_{k}\left(t, C_{j}\right)+N\left(\tilde{x}_{k}\left(t, C_{j}\right)\right)+g_{k}\left(\tilde{x}_{k}\left(t, C_{j}\right)\right)=0 \forall k=1,2 \cdots m$,

\section{Numerical Examples and Results}

In this section, three examples are presented to illustrate the efficiency of the new method for solving NLPCOPs. The calculations are performed using maple software 2018, HP ENVY laptop 13 corei7 8th Gen 16GB.

Example 1. Consider the NLPCOP test problem from Schittkowski [54] (No. 216).

$$
\begin{array}{ll}
\text { Minimize } & f(x)=100\left(x_{1}^{2}-x_{2}\right)^{2}+\left(x_{1}-1\right)^{2}, \\
\text { subject to } & h(x)=x_{1}\left(x_{1}-4\right)-2 x_{2}+12=0,
\end{array}
$$

whose exact solution is not known, but expected optimal solution is $x_{1}^{*}=1.9993, x_{2}^{*}=3.9998$. First, we transform the constraint problem to an unconstrained problem by quadratic penalty function for $\sigma=2$; then, we have

$$
\begin{aligned}
f(x, \mu)= & 100\left(x_{1}^{2}-x_{2}\right)^{2}+\left(x_{1}-1\right)^{2} \\
& +\frac{1}{2} \mu\left(x_{1}\left(x_{1}-4\right)-2 x_{2}+12\right)^{2},
\end{aligned}
$$

where $\mu \in \mathfrak{R}^{+}$, and so that the nonlinear FOGBDS can be given as

$$
\begin{gathered}
T^{\alpha} x_{1}(t)=-400\left(x_{1}^{2}-x_{2}\right) x_{1}-2\left(x_{1}-1\right) \\
-\mu\left(2 x_{1}-4\right)\left(x_{1}^{2}-4 x_{1}-2 x_{2}+12\right), \\
T^{\alpha} x_{2}(t)=200\left(x_{1}^{2}-x_{2}\right)+2 \mu\left(x_{1}^{2}-4 x_{1}-2 x_{2}+12\right), \\
x_{1}(0)=0, x_{2}(0)=0,
\end{gathered}
$$

where $0<\alpha \leq 1$. By using OHAM-LS with auxiliary penalty variable $\mu=200$, the terms of the OHAM-LS solutions for fractional order are acquired by using the concept of homotopy. According to Equation (6)), we choose the linear and nonlinear operators in the following forms:

$$
\begin{aligned}
L_{1}\left[\varphi_{1}(t, p)\right]= & T^{\alpha} \varphi_{1}(t, p), \\
L_{2}\left[\varphi_{2}(t, p)\right]= & T^{\alpha} \varphi_{2}(t, p), \\
N_{1}\left[\varphi_{1}(t, p)\right]= & T^{\alpha} \varphi_{1}(t, p)+400\left(\varphi_{1}(t, p)^{2}-\varphi_{2}(t, p)\right) \varphi_{1}(t, p) \\
& +2\left(\varphi_{1}(t, p)-1\right)+200\left(2 \varphi_{1}(t, p)-4\right) \\
& \cdot\left(\varphi_{1}(t, p)^{2}-4 \varphi_{1}(t, p)-2 \varphi_{2}(t, p)+12\right), \\
N_{2}\left[\varphi_{2}(t, p)\right]= & T^{\alpha} \varphi_{2}(t, p)-200\left(\varphi_{1}(t, p)^{2}-\varphi_{2}(t, p)\right) \\
& -400\left(\varphi_{1}(t, p)^{2}-4 \varphi_{1}(t, p)-2 \varphi_{2}(t, p)+12\right) .
\end{aligned}
$$


We can construct the following homotopy

$$
\begin{aligned}
(1-p) T^{\alpha} \varphi_{1}(t, p)= & H(p)\left[T^{\alpha} \varphi_{1}(t, p)+400\left(\varphi_{1}(t, p)^{2}\right.\right. \\
& \left.-\varphi_{2}(t, p)\right) \varphi_{1}(t, p)+2\left(\varphi_{1}(t, p)-1\right) \\
& +200\left(2 \varphi_{1}(t, p)-4\right)\left(\varphi_{1}(t, p)^{2}\right. \\
& \left.\left.-4 \varphi_{1}(t, p)-2 \varphi_{2}(t, p)+12\right)\right] \\
(1-p) T^{\alpha} \varphi_{2}(t, p)= & H(p)\left[T^{\alpha} \varphi_{2}(t, p)-200\left(\varphi_{1}(t, p)^{2}\right.\right. \\
& \left.-\varphi_{2}(t, p)\right)-400\left(\varphi_{1}(t, p)^{2}-4 \varphi_{1}(t, p)\right. \\
& \left.\left.-2 \varphi_{2}(t, p)+12\right)\right],
\end{aligned}
$$

where

$$
\begin{aligned}
\varphi_{1}(t, p) & =x_{1,0}(t)+\sum_{j \leq 1} x_{1, j}(t) p^{j}, \\
\varphi_{2}(t, p) & =x_{2,0}(t)+\sum_{j \leq 1} x_{2, j}(t) p^{j}, \\
H_{k}(p) & =p C_{1}+p^{2} C_{2}+p^{3}+C_{3}+\cdots, \quad k=1,2 \cdots m .
\end{aligned}
$$

Substituting Equations (56)-(58) into Equations (54) and (55) and equating the coefficient of the same powers of $p$ result to the following set of linear FDEs.

$$
\begin{aligned}
p^{0}: T^{\alpha} x_{1,0}(t)= & 0 \\
p^{0}: T^{\alpha} x_{2,0}(t)= & 0 \\
p^{1}: T^{\alpha} x_{1,1}(t)= & 2000 x_{1,0}^{3} C_{1}+T^{\alpha} x_{1,0} C_{1}-3600 x_{2,0} x_{1,0} C_{1} \\
& -9600 x_{1,0}^{2} C_{1}-T^{\alpha} x_{1,0}+6400 x_{2,0} C_{1} \\
& +32002 x_{1,0} C_{1}-38402 C_{1}=0 \\
p^{1}: T^{\alpha} x_{2,1}(t)= & T^{\alpha} x_{2,0} C_{1}-1800 x_{1,0}^{2} C_{1}-T^{\alpha} x_{2,0} \\
& +6400 x_{1,0} C_{1}+3400 x_{2,0} C_{1}-19200 C_{1}=0
\end{aligned}
$$

$$
\begin{aligned}
p^{2}: T^{\alpha} x_{1,2}(t)= & 2000 x_{1,0}^{3} C_{2}+6000 x_{1,0}^{2} x_{1,1} C_{1} \\
& +T^{\alpha} x_{1,0} C_{2}+T^{\alpha} x_{1,1} C_{1}-3600 x_{2,0} x_{1,0} C_{2} \\
& -3600 x_{2,0} x_{1,1} C_{1}-9600 x_{1,0}^{2} C_{2} \\
& -19200 x_{1,0} x_{1,1} C_{1}-3600 x_{1,0} x_{2,1} C_{1} \\
& -T^{\alpha} x_{1,1}+6400 x_{2,0} C_{2}+32002 x_{1,0} C_{2} \\
& +32002 x_{1,1} C_{1}+6400 x_{2,1} C_{1}-38402 C_{2}=0,
\end{aligned}
$$

$$
\begin{aligned}
p^{2}: T^{\alpha} x_{2,2}(t)= & T^{\alpha} x_{2,0} C_{2}+T^{\alpha} x_{2,1} C_{1}-1800 x_{1,0}^{2} C_{2} \\
& -3600 x_{1,0} x_{1,1} C_{1}-T^{\alpha} x_{2,1}+6400 x_{1,0} C_{2} \\
& +6400 x_{1,1} C_{1}+3400 x_{2,0} C_{2}+3400 x_{2,1} C_{1} \\
& -19200 C_{2}=0 .
\end{aligned}
$$

Applying the operator $I^{\alpha}$ to both sides of Equations (59)(64) with initial conditions given in Equation (5.6), we obtain

$$
\begin{aligned}
& x_{1,0}(t)=0 \\
& x_{2,0}(t)=0, \\
& x_{1,1}\left(t, C_{1}\right)=384020 t^{1 / 10} C_{1}, \\
& x_{2,1}\left(t, C_{1}\right)=192000 t^{1 / 10} C_{1}, \\
& x_{1,2}\left(t, C_{1}, C_{2}\right)=-6.759104020 \times 10^{10} t^{1 / 5} C_{1}^{2} \\
&-384020 t^{1 / 10}+384020 t^{1 / 10} C_{1} \\
&+384020 C_{2} t^{1 / 10}, \\
& x_{2,2}\left(t, C_{1}, C_{2}\right)=-1.555264000 \times 10^{10} t^{1 / 5} C_{1}^{2} \\
&-192000 t^{1 / 10} C_{1}^{2}+192000 t^{1 / 10} C_{1} \\
&+192000 C_{2} t^{1 / 10} .
\end{aligned}
$$

Adding up the solution components Equations (65)-(70), the 2nd-order approximate solution obtained by OHAM-LS at $\alpha=0.9$, for $p=1$, are

$$
\begin{aligned}
x_{1}^{2}\left(t, C_{1}, C_{2}\right)= & \left(768040 C_{1}-384020 C_{1}^{2}+384020 C_{2}\right) t^{1 / 10} \\
& -6.759104020 \times 10^{10} t^{1 / 5} C_{1}^{2}, \\
x_{2}^{2}\left(t, C_{1}, C_{2}\right)= & \left(384000 C_{1}-192000 C_{1}^{2}+192000 C_{2}\right) t^{1 / 10} \\
& -1.555264000 \times 10^{10} t^{1 / 5} C_{1}^{2} .
\end{aligned}
$$

For the calculations of $C_{1}$ and $C_{2}$ in $x_{1}^{2}(t)$ and $x_{2}^{2}(t)$ given in Equations (71) and (72), we apply the procedure mentioned in Equations (19)-(21); we obtain, for $x_{1}^{2}(t)$,

$$
\begin{aligned}
& c[1]=1.800506863 \times 10^{-6}, \\
& c[2]=6.594892833 \times 10^{-6},
\end{aligned}
$$

and for $x_{2}^{2}(t)$,

$$
\begin{aligned}
& c[1]=0.111906918 \times 10^{-4}, \\
& c[2]=0.2190543167 \times 10^{-4} .
\end{aligned}
$$

Substituting these optimal values into Equations (71) and (72) becomes

$$
\begin{aligned}
& x_{1}^{2}(t)=4.196444315 t^{1 / 10}-1.084631569 t^{1 / 5}, \\
& x_{2}^{2}(t)=7.546421106 t^{1 / 10}-0.7996784175 t^{1 / 5} .
\end{aligned}
$$

Table 1 shows the $C_{k}$ at different values of $\alpha$ for Example 1. Table 2 shows the comparisons and the absolute error between OHAM-LS and RK4 at different values of $\alpha=1$. Figure 1 shows the analytical approximate solutions obtained by OHAM-LS for $\alpha=1,0.9,0.8$, and 0.7 with RK 4 at $\alpha=1$. 
TABLE 1: Control-convergence parameters $C_{k}$ at different values of $\alpha$.

\begin{tabular}{lcccc}
\hline Variable & $x_{1}(t)$ & $x_{1}(t)$ & $x_{2}(t)$ & $x_{2}(t)$ \\
$\alpha$ & $C_{1}$ & $C_{2}$ & $C_{1}$ & $C_{2}$ \\
\hline 1 & $1.912514527 \times 10^{-6}$ & $7.294797236 \times 10^{-6}$ & $0.113698734 \times 10^{-4}$ & $0.229361274 \times 10^{-4}$ \\
0.9 & $1.800506863 \times 10^{-6}$ & $6.594892833 \times 10^{-6}$ & $0.111906918 \times 10^{-4}$ & $0.2190543167 \times 10^{-4}$ \\
0.8 & $1.714313871 \times 10^{-6}$ & $5.524430129 \times 10^{-6}$ & $0.10992623 \times 10^{-4}$ & $0.201017632 \times 10^{-4}$ \\
0.7 & $1.593611093 \times 10^{-6}$ & $5.294592861 \times 10^{-6}$ & $0.107191284 \times 10^{-4}$ & $0.197911283 \times 10^{-4}$ \\
\hline
\end{tabular}

TABLe 2: Comparisons and absolute error between OHAM-LS and RK4, $\alpha=1$.

\begin{tabular}{lcccccc}
\hline$t_{k}$ & OHAM-LS $x_{1}(t)$ & OHAM-LS $x_{2}(t)$ & RK4 $x_{1}(t)$ & RK4 $x_{2}(t)$ & Error $x_{1}(t)$ & Error $x_{2}(t)$ \\
\hline 0.0000 & 0.000000 & 0.000000 & 0.000000 & 0.000000 & 0.000000 & 0.000000 \\
0.0005 & 1.970211 & 3.871721 & 1.970899 & 3.871887 & 0.000688 & 0.000166 \\
0.0010 & 1.977134 & 3.907823 & 1.978274 & 3.907993 & 0.001136 & 0.00017 \\
0.0013 & 1.981102 & 3.922403 & 1.981384 & 3.922554 & 0.000282 & 0.000151 \\
0.0015 & 1.982211 & 3.930534 & 1.983132 & 3.930578 & 0.000921 & $4.4 E-05$ \\
0.0020 & 1.983214 & 3.935653 & 1.984252 & 3.935654 & 0.001038 & $1 E-06$ \\
\hline
\end{tabular}

Example 2. Consider the NLPCOPs test problem from Schittkowski [54] [No 320].

$$
\begin{array}{ll}
\text { Minimize } & f(x)=\left(x_{1}-20\right)^{2}+\left(x_{2}+20\right)^{2} \\
\text { subject to } & h(x)=\frac{x_{1}^{2}}{100}+\frac{x_{2}^{2}}{4}-1=0 .
\end{array}
$$

This is a practical problem, and the exact solution is not known, but the expected optimal solution is $x_{1}^{*}=9.395, x_{2}^{*}$ $=-0.6846$. First, the quadratic penalty function is used to get the unconstrained optimization problem as follows:

$$
F(x, \mu)=\left(x_{1}-20\right)^{2}+\left(x_{2}+20\right)^{2}+\frac{1}{2} \mu\left(\frac{x_{1}^{2}}{100}+\frac{x_{2}^{2}}{4}-1\right)^{2},
$$

where $\mu \in \mathfrak{R}^{+}$and so that the nonlinear FOGBDS be given as

$$
\begin{aligned}
T^{\alpha} x_{1}(t)=2 x_{1}-40+\mu & \left(\frac{1}{5000} x_{1}^{3}+\frac{1}{200} x_{1} x_{2}^{2}-\frac{1}{50} x_{1}\right) \\
T^{\alpha} x_{2}(t)=2 x_{2}+40+\mu & \left(\frac{1}{200} x_{2} x_{1}^{2}+\frac{1}{8} x_{2}^{3}-\frac{1}{2} x_{2}\right) \\
0 & <\alpha \leq 1, x_{1}(0)=0, x_{2}(0)=0 .
\end{aligned}
$$

By using OHAM-LS with $\mu=10^{6}$, the terms of the OHAM-LS solutions for fractional order are acquired by using the concept of homotopy. According to Equation (6), we choose the linear and nonlinear operators in the following forms:

$$
L_{1}\left[\varphi_{1}(t, p)\right]=T^{\alpha} \varphi_{1}(t, p),
$$

$$
L_{2}\left[\varphi_{2}(t, p)\right]=T^{\alpha} \varphi_{2}(t, p),
$$

$$
\begin{aligned}
N_{1}\left[\varphi_{1}(t, p)\right]= & T^{\alpha} \varphi_{1}(t, p)-2\left(\varphi_{1}(t, p)+40\right. \\
& -10^{6}\left(\frac{1}{5000} \varphi_{1}(t, p)^{3}\right. \\
& \left.\left.+\frac{1}{200} \varphi_{1}(t, p) \varphi_{2}(t, p)^{2}-\frac{1}{50} \varphi_{1}(t, p)\right)\right),
\end{aligned}
$$

$$
\begin{aligned}
N_{2}\left[\varphi_{2}(t, p)\right]= & T^{\alpha} \varphi_{2}(t, p)-2 \varphi_{2}(t, p)-40 \\
& -10^{6}\left(\frac{1}{200} \varphi_{2}(t, p) \times \varphi_{1}(t, p)^{2}\right. \\
& \left.-\frac{1}{8} \varphi_{2}(t, p)^{3}+\frac{1}{2} \varphi_{2}(t, p)\right),
\end{aligned}
$$

$$
\begin{aligned}
(1-p) T^{\alpha} \varphi_{1}(t, p)= & H(p)\left[T^{\alpha} \varphi_{1}(t, p)-2\left(\varphi_{1}(t, p)+40\right.\right. \\
& -10^{6}\left(\frac{1}{5000} \varphi_{1}(t, p)^{3}+\frac{1}{200} \varphi_{1}(t, p)\right. \\
& \left.\left.\times \varphi_{2}(t, p)^{2}-\frac{1}{50} \varphi_{1}(t, p)\right)\right],
\end{aligned}
$$

$$
\begin{aligned}
(1-p) T^{\alpha} \varphi_{2}(t, p)= & H(p)\left[T^{\alpha} \varphi_{2}(t, p)-2 \varphi_{2}(t, p)-40\right. \\
& -10^{6}\left(\frac{1}{200} \varphi_{2}(t, p) \times \varphi_{1}(t, p)^{2}\right. \\
& \left.\left.-\frac{1}{8} \varphi_{2}(t, p)^{3}+\frac{1}{2} \varphi_{2}(t, p)\right)\right],
\end{aligned}
$$




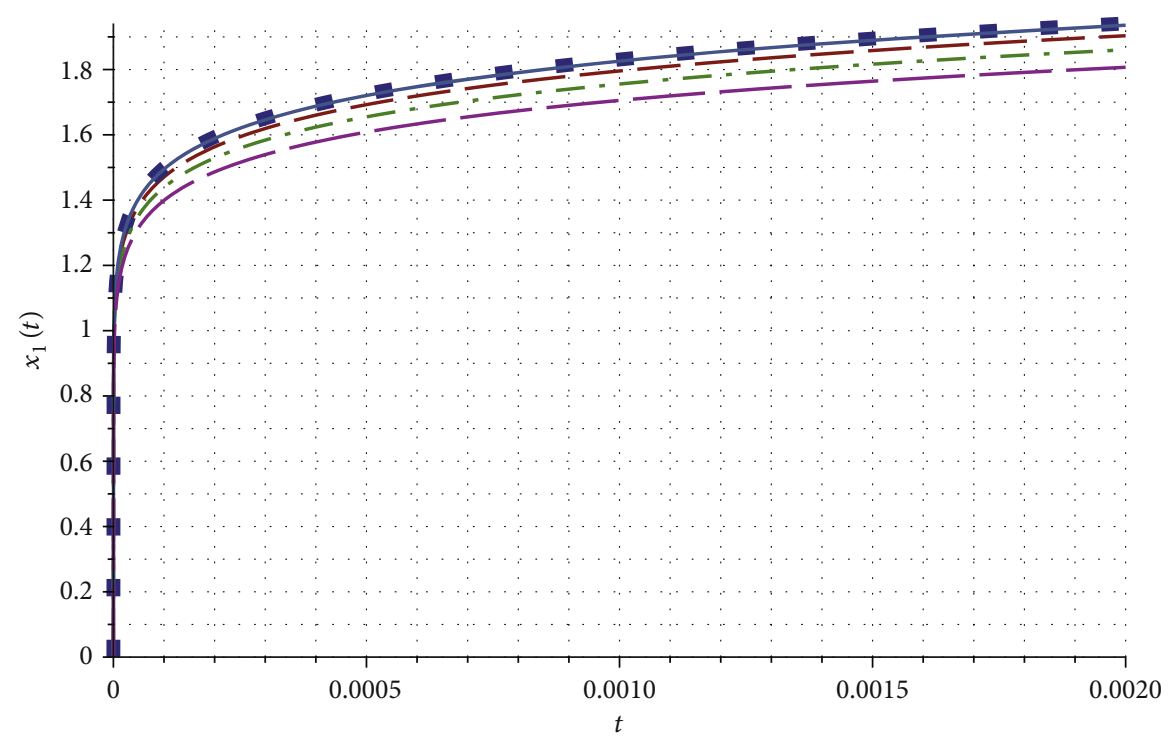

(a)

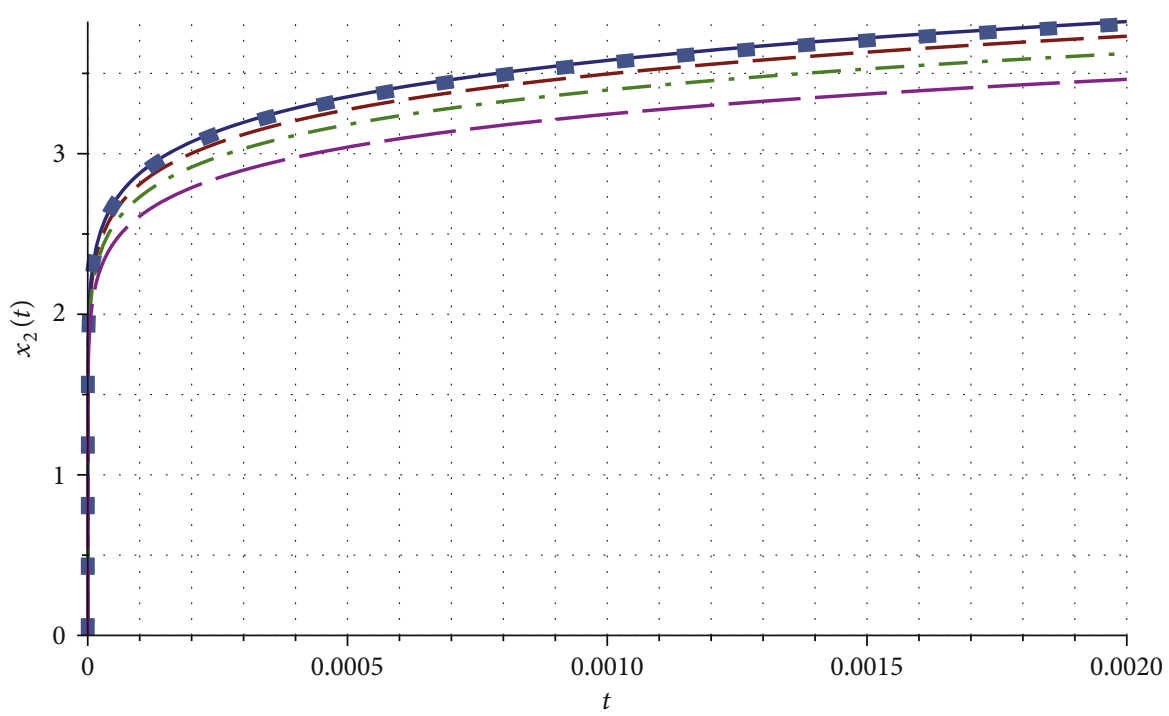

(b)

Figure 1: (a) Different values of $\alpha$ (OHAM-LS; $\alpha=1$, dot; $\alpha=0.9$, dash; $\alpha=0.8$, dash dot; and $\alpha=0.7$, long dash) and Rk4 ( $\alpha=1$, solid) at $x_{1}$. (b) Different values of $\alpha$ (OHAM-LS; $\alpha=1$, dot; $\alpha=0.9$, dash; $\alpha=0.8$, dash dot; and $\beta=0.7$, long dash) and RK4 ( $\alpha=1$, solid) at $x_{2}$.

where

$$
\begin{aligned}
\varphi_{1}(t, p) & =x_{1,0}(t)+\sum_{j \leq 1} x_{1, j}(t) p^{j}, \\
\varphi_{2}(t, p) & =x_{2,0}(t)+\sum_{j \leq 1} x_{2, j}(t) p^{j}, \\
H_{k}(p) & =p C_{1}+p^{2} C_{2}+p^{3}+C_{3}+\cdots, \quad k=1,2 .
\end{aligned}
$$

$$
p^{0}: T^{\alpha} x_{2,0}(t)=0
$$

Substituting Equations (85)-(87) into Equations (83) and (84) and equating the coefficient of the same powers of $p$ yields the following set of linear FDEs:

$$
p^{0}: T^{\alpha} x_{1,0}(t)=0
$$

$$
\begin{aligned}
p^{1}: T^{\alpha} x_{1,1}(t)= & -200 x_{1,0}^{3} C_{1}-5000 x_{1,0} x_{2,0}^{2} C_{1} \\
& +T^{\alpha} x_{1,0} C_{1}-T^{\alpha} x_{1,0}+19998 x_{1,0} C_{1} \\
& +40 C_{1},
\end{aligned}
$$

$$
\begin{aligned}
p^{1}: T^{\alpha} x_{2,1}(t)= & -125000 x_{2,0}^{3} C_{1}-5000 x_{2,0} x_{1,0}^{2} C_{1} \\
& -T^{\alpha} x_{2,0} C_{1}-2 x_{2,0} C_{1}-T^{\beta} x_{2,0} \\
& +500000 x_{1,0} C_{1}-40 C_{1}=0,
\end{aligned}
$$


TABLE 3: Control-convergence parameters $C_{k}$ at different values of $\alpha$.

\begin{tabular}{lcccc}
\hline Variable & $x_{1}(t)$ & $x_{1}(t)$ & $x_{2}(t)$ & $x_{2}(t)$ \\
$\alpha$ & $C_{1}$ & $C_{2}$ & $C_{1}$ & $C_{2}$ \\
\hline 1 & $-0.1198434251 \times 10^{-3}$ & -0.02645325610 & $-1.3256727843 \times 10^{-5}$ & $-2.527402984 \times 10^{-3}$ \\
0.9 & $-0.1208162856 \times 10^{-3}$ & -0.02826592550 & $-1.343994006 \times 10^{-5}$ & $-2.536649902 \times 10^{-3}$ \\
0.8 & $-0.1487623674 \times 10^{-3}$ & -0.02983123651 & $-1.3619012564 \times 10^{-5}$ & $-2.550122356 \times 10^{-3}$ \\
0.7 & $-0.1598723560 \times 10^{-3}$ & -0.03154109428 & $-1.3801234527 \times 10^{-5}$ & $-2.573641295 \times 10^{-3}$ \\
\hline
\end{tabular}

TABLE 4: Comparison and absolute error between OHAM-LS and RK4, $\alpha=1$.

\begin{tabular}{lcccccc}
\hline$t_{k}$ & OHAM-LS $x_{1}(t)$ & OHAM-LS $x_{2}(t)$ & RK4 $x_{1}(t)$ & RK4 $x_{2}(t)$ & Error $x_{1}(t)$ & Error $x_{2}(t)$ \\
\hline 0.0 & 0.000000 & 0.000000 & 0.000000 & 0.000000 & 0.000000 & 0.000000 \\
1.0 & 5.847225 & -0.473547 & 5.847465 & -0.477547 & 0.00024 & -0.004 \\
2.0 & 6.741648 & -0.542275 & 6.741904 & -0.542553 & 0.000256 & -0.000278 \\
4.0 & 8.455454 & -0.584110 & 8.457551 & -0.584133 & 0.002097 & $-2.3 E-05$ \\
6.0 & 8.731031 & -0.615205 & 8.732010 & -0.615305 & 0.000979 & $-1 E-04$ \\
7.0 & 8.977101 & -0.640324 & 8.977187 & -0.640466 & $8.6 E-05$ & -0.000142 \\
8.0 & 9.090736 & -0.661201 & 9.090838 & -0.661673 & 0.000102 & -0.000472 \\
10.0 & 9.303112 & -0.680032 & 9.303232 & -0.680066 & 0.00012 & $-3.4 E-05$ \\
\hline
\end{tabular}

$$
\begin{aligned}
p^{2}: T^{\alpha} x_{1,2}(t)= & -200 x_{1,0}^{3} C_{2}-6000 x_{1,0}^{2} x_{1,1} C_{1} \\
& -5000 x_{1,0} x_{2,0}^{2} C_{2}-10000 x_{1,0} x_{2,0} x_{2,1} C_{1} \\
& -5000 x_{2,0}^{2} x_{1,1} C_{1}+T^{\alpha} x_{1,0} C_{2}+T^{\alpha} x_{1,1} C_{1} \\
& -T^{\alpha} x_{1,1}+19998 x_{1,0} C_{2}+19998 x_{1,1} C_{1} \\
& +40 C_{2}=0,
\end{aligned}
$$

$$
\begin{aligned}
p^{2}: T^{\alpha} x_{2,2}(t)= & -125000 x_{2,0}^{3} C_{2}-375000 x_{2,0}^{2} x_{2,1} C_{1} \\
& -10000 x_{2,0} x_{1,1} x_{1,0} C_{1}-5000 x_{2,0} x_{1,0}^{2} C_{2} \\
& -5000 x_{2,1} x_{1,0}^{2} C_{1}+T^{\alpha} x_{2,1} C_{1}+T^{\alpha} x_{2,0} C_{2} \\
& -2 x_{2,0} C_{2}-2 x_{2,1} C_{1}+500000 x_{1,1} C_{1} \\
& -T^{\alpha} x_{2,1}+500000 x_{1,0} C_{2}-40 C_{2}=0 .
\end{aligned}
$$

Applying the operator $I^{\alpha}$ on both sides of Equations (88)(93) with initial conditions given in Equation (4.38), we obtain

$$
\begin{aligned}
x_{1,0}(t)= & , \\
x_{2,0}(t)= & , \\
x_{1,1}\left(t, C_{1}\right)= & -200 t^{1 / 5} C_{1}, \\
x_{2,1}\left(t, C_{1}\right)= & 200 t^{1 / 5} C_{1}, \\
x_{1,2}\left(t, C_{1}, C_{2}\right)= & 9.999000 \times 10^{6} t^{2 / 5} C_{1}^{2}+200 t^{1 / 5} C_{1}^{2} \\
& -200 t^{1 / 5} C_{1}-200 C_{2} t^{1 / 5}, \\
x_{2,2}\left(t, C_{1}, C_{2}\right)= & 2.50001000 \times 10^{8} t^{2 / 5} C_{1}^{2}-200 t^{1 / 5} C_{1}^{2} \\
& +200 t^{1 / 5} C_{1}+200 C_{2} t^{1 / 10} .
\end{aligned}
$$

Adding up the solution components Equations (94)-(99), the 2nd-order approximate solution obtained by OHAM-LS at $\alpha=0.9$, for $p=1$, is

$$
x_{1}^{2}(t)=t^{1 / 5}\left(-400 C_{1}-9.999000 \times 10^{6} t^{1 / 5} C_{1}^{2}+200 C_{1}^{2}-200 C_{2}\right),
$$

$x_{2}^{2}(t)=t^{1 / 5}\left(400 C_{1}-2.50001000 \times 10^{8} t^{1 / 5} C_{1}^{2}-200 C_{1}^{2}+200 C_{2}\right)$.

For the calculations of $C_{1}$ and $C_{2}$ in $x_{1}^{2}(t)$ and $x_{2}^{2}(t)$ given in Equations (100) and (101), we apply the procedure mentioned in Equations (19)-(21), we obtain for $x_{1}^{2}(t)$,

$$
\begin{aligned}
& c[1]=-0.1208162856 \times 10^{-3}, \\
& c[2]=-0.02826592550 .
\end{aligned}
$$

And for $x_{2}^{2}(t)$,

$$
\begin{aligned}
& c[1]=-1.343994006 \times 10^{-5}, \\
& c[2]=-2.536649902 \times 10^{-3} .
\end{aligned}
$$

Substituting these optimal values into Equations (100) and (101), we have

$$
\begin{aligned}
& x_{1}^{2}(t)=\left(5.701514534+0.1459511521 t^{1 / 5}\right) t^{1 / 5}, \\
& x_{2}^{2}(t)=\left(-0.5167059926+0.04515817783 t^{1 / 5}\right) t^{1 / 5} .
\end{aligned}
$$

Table 3 shows the $C_{k}$ at different values of $\alpha$ for example 2. Table 4 show the comparisons and the absolute error between OHAM-LS and RK4 at different values of $\alpha=1$. 


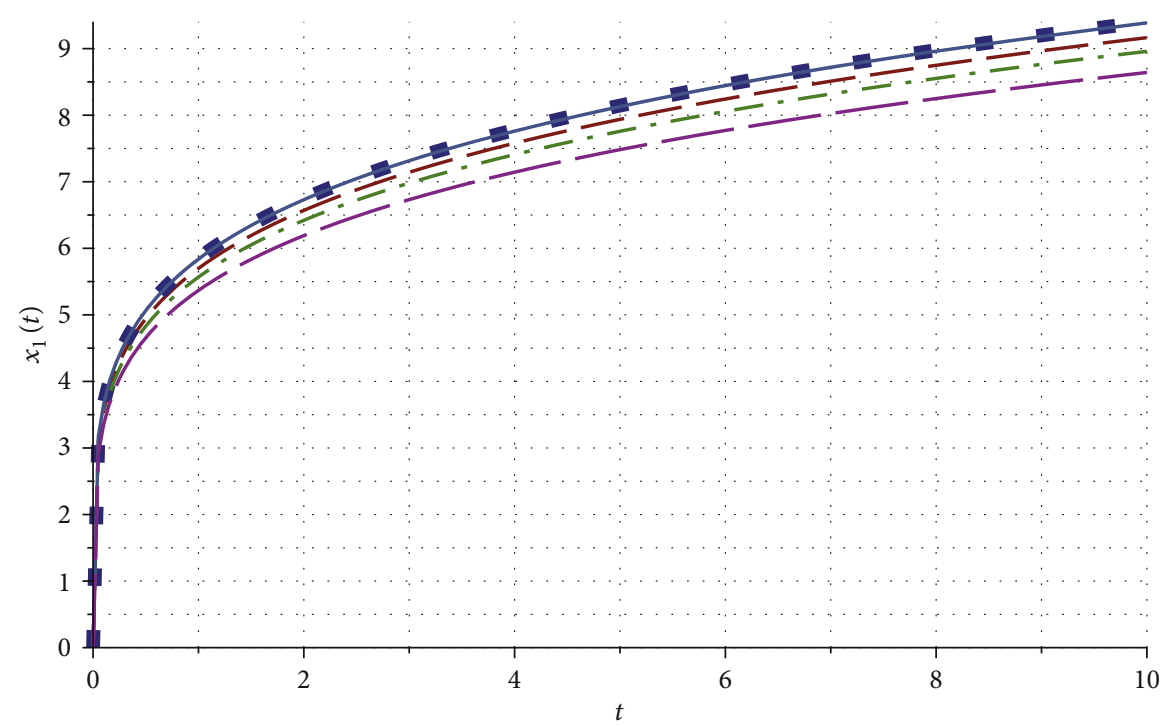

(a)

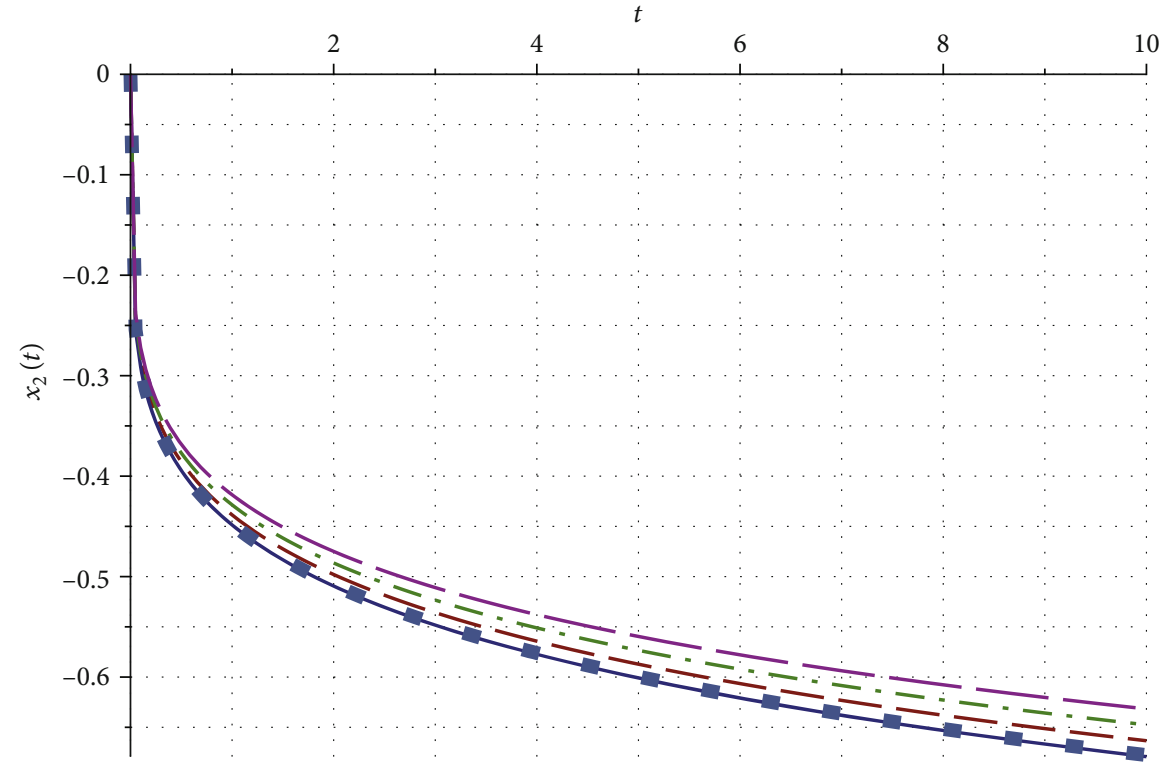

(b)

Figure 2: (a) Different values of $\alpha$ (OHAM-LS; $\alpha=1$ dot, $\alpha=0.9$ dash, $\alpha=0.8$ dash dot, and $\alpha=0.7$ long dash) and Rk4 ( $\alpha=1$, solid) at $x_{1}$. (b) Different values of $\alpha$ (OHAM-LS; $\alpha=1$ dot, $\alpha=0.9$ dash, $\alpha=0.8$ dash dot, and $\alpha=0.7$ long dash) and RK 4 ( $\alpha=1$, solid) at $x_{2}$.

Figure 2 show the analytical approximate solutions obtained by OHAM-LS for $\alpha=1,0.9,0.8$, and 0.7 with RK4 at $\alpha=1$.

Example 3. Consider the NLPCOP test problem from Schittkowski [54] (No. 300).

$$
\begin{array}{ll}
\text { Minimize } & f(x)=x_{1}^{2}+x_{2}^{2}+2 x_{3}^{2}+x_{4}^{2}-5 x_{1}-5 x_{2}-21 x_{3}+7 x_{4}, \\
\text { subject to } & 8-x_{1}^{2}-x_{2}^{2}-x_{3}^{2}-x_{4}^{2}-x_{1}+x_{2}-x_{3}+x_{4} \leq 0 \\
& 10-x_{1}^{2}-2 x_{2}^{2}-x_{3}^{2}+x_{1}+x_{4} \leq 0 \\
& 5-2 x_{1}^{2}-x_{2}^{2}-x_{3}^{2}-2 x_{1}+x_{2}+x_{4} \leq 0
\end{array}
$$

This is a practical problem, and the exact solution is not known, but the expected optimal solution is $x_{1}^{*}=0, x_{2}^{*}=1$, $x_{3}^{*}=2$, and $x_{4}^{*}=-1$. From the above procedure, the secondorder approximate solution obtained by OHAM-LS at $\alpha=$ 0.9 , for $p=1$, is

$$
\begin{aligned}
x_{1}^{2}(t)= & \left(16100 C_{1}-8050 C_{1}^{2}+8050 C_{2}\right) t^{1 / 10} \\
& +1.54569500 \times 10^{8} t^{1 / 5} C_{1}^{2}, \\
x_{2}^{2}(t)= & \left(-25900 C_{1}+12950 C_{1}^{2}-12950 C_{2}\right) t^{1 / 10} \\
& -3.75020500 \times 10^{8} t^{1 / 5} C_{1}^{2},
\end{aligned}
$$


TABLE 5: Control-convergence parameters $C_{k}$ at different values of $\alpha$.

\begin{tabular}{lccc}
\hline Variable & $x_{2}(t)$ & $x_{2}(t)$ & $x_{3}(t)$ \\
$\alpha$ & $C_{1}$ & $C_{2}$ & $C_{1}$ \\
\hline 1 & $-4.470470112 \times 10^{-13}$ & $-0.1542801253 \times 10^{-3}$ & $-1.0784243190 \times 10^{-4}$ \\
0.9 & $-4.494712729 \times 10^{-13}$ & $-0.1618198317 \times 10^{-3}$ & $-1.096696787 \times 10^{-4}$ \\
0.8 & $-4.5167327196 \times 10^{-13}$ & $-0.1832920121 \times 10^{-3}$ & $-1.1079094521 \times 10^{-4}$ \\
0.7 & $-4.5371220162 \times 10^{-13}$ & $-0.2087212810 \times 10^{-3}$ & $-1.1261409123 \times 10^{-4}$ \\
\hline
\end{tabular}

TABLE 6: Control-convergence parameters $C_{k}$ at different values of $\alpha$.

\begin{tabular}{lccr}
\hline Variable & $x_{3}(t)$ & $x_{4}(t)$ & $x_{4}(t)$ \\
$\alpha$ & $C_{2}$ & $C_{1}$ & $C_{2}$ \\
\hline 1 & $-0.5711237191 \times 10^{-3}$ & $-5.921274832 \times 10^{-12}$ & $0.8973526178 \times 10^{-4}$ \\
0.9 & $0.599293243 \times 10^{-3}$ & $-5.935109529 \times 10^{-12}$ & $0.8773054262 \times 10^{-4}$ \\
0.8 & $-0.6190253261 \times 10^{-3}$ & $-5.953158452 \times 10^{-12}$ & $0.8696526178 \times 10^{-4}$ \\
0.7 & $-0.62131534211 \times 10^{-3}$ & $-5.975232801 \times 10^{-12}$ & $0.8572034710 \times 10^{-4}$ \\
\hline
\end{tabular}

TABLE 7: Comparisons and absolute error between OHAM-LS and RK4, $\alpha=1$.

\begin{tabular}{lcccc}
\hline \multirow{2}{*}{$t_{k}$} & $\begin{array}{c}\text { OHAM- } \\
\text { LS } x_{2}(t)\end{array}$ & $\begin{array}{c}\text { OHAM- } \\
\text { LS } x_{3}(t)(t)\end{array}$ & $\begin{array}{c}\text { OHAM- } \\
\text { LS } x_{4}(t)\end{array}$ & RK4 $x_{2}(t)$ \\
\hline 0.000 & 0.000000 & 0.000000 & 0.000000 & 0.000000 \\
0.001 & 0.834165 & 1.835129 & -0.805509 & 0.834260 \\
0.002 & 0.894101 & 1.886099 & -0.859211 & 0.894137 \\
0.003 & 0.931112 & 1.928753 & -0.963765 & 0.931137 \\
0.004 & 0.958211 & 1.965657 & -0.978625 & 0.958313 \\
0.005 & 0.979623 & 1.998334 & -0.991899 & 0.979937 \\
\hline
\end{tabular}

$$
\begin{aligned}
x_{3}^{2}(t)= & \left(16420 C_{1}-8210 C_{1}^{2}+8210 C_{2}\right) t^{1 / 10} \\
& +1.62525800 \times 10^{8} t^{1 / 5} C_{1}^{2} \\
x_{4}^{2}(t)= & \left(-46140 C_{1}+23070 C_{1}^{2}-23070 C_{2}\right) t^{1 / 10} \\
& -1.24619300 \times 10^{8} t^{1 / 5} C_{1}^{2} .
\end{aligned}
$$

For the calculations of $C_{1}$ and $C_{2}$ in $x_{1}^{2}(t), x_{2}^{2}(t), x_{3}^{2}(t)$, and $x_{4}^{2}(t)$ given in Equations (4.79)-(4.82), we apply the procedure mentioned in Equations (19)-.(21); we obtain for $x_{1}^{2}(t)$,

$$
c[1]=0, c[2]=0
$$

and for $x_{2}^{2}(t)$,

$$
\begin{aligned}
& c[1]=-4.494712729 \times 10^{-13}, \\
& c[2]=-0.1618198317 \times 10^{-4},
\end{aligned}
$$

and for $x_{3}^{2}(t)$,

$$
\begin{aligned}
& c[1]=-1.096696787 \times 10^{-14}, \\
& c[2]=0.599293243 \times 10^{-3},
\end{aligned}
$$

TABLE 8: Comparison and absolute error between OHAM-LS and RK4, $\alpha=1$.

\begin{tabular}{lccccc}
\hline$t_{k}$ & $\mathrm{RK} 4 x_{3}(t)$ & $\mathrm{RK} 4 x_{4}(t)$ & Error $x_{2}(t)$ & Error $x_{3}(t)$ & Error $x_{4}(t)$ \\
\hline 0.000 & 0.000000 & 0.000000 & 0.000000 & 0.000000 & 0.000000 \\
0.001 & 1.835278 & -0.805746 & $9.5 \mathrm{E}-05$ & 0.000149 & -0.000237 \\
0.002 & 1.886124 & -0.859310 & $3.6 \mathrm{E}-05$ & $2.5 \mathrm{E}-05$ & $-9.9 \mathrm{E}-05$ \\
0.003 & 1.928838 & -0.963858 & $2.5 \mathrm{E}-05$ & $8.5 \mathrm{E}-05$ & $-9.3 \mathrm{E}-05$ \\
0.004 & 1.965795 & -0.978831 & 0.001372 & 0.000138 & -0.000206 \\
0.005 & 1.998449 & -0.991990 & 0.000314 & 0.000115 & $-9.1 \mathrm{E}-05$ \\
\hline
\end{tabular}

and for $x_{4}^{2}(t)$,

$$
\begin{aligned}
& c[1]=-5.935109529 \times 10^{-12}, \\
& c[2]=0.8773054262 \times 10^{-4} .
\end{aligned}
$$

Substituting these optimal values into Equations (106)(109), we have

$$
\begin{aligned}
& \tilde{x}_{1}(t)=0, \\
& \tilde{x}_{2}(t)=0.095566833 \times t^{1 / 10}-7.576330095 \times 10^{-17} t^{1 / 5}, \\
& \tilde{x}_{3}(t)=3.119322656 \times t^{1 / 10}+1.954769053 \times t^{1 / 5}, \\
& \tilde{x}_{4}(t)=-2.023943344 \times t^{1 / 10}-4.389780283 \times 10^{-15} t^{1 / 5} .
\end{aligned}
$$

Tables 5 and 6 show the $C_{k}$ at different values of $\alpha$ for Example 3. Tables 7 and 8 show the comparisons and the absolute error between OHAM-LS and RK4 at $\alpha=1$. Also, Figure 3 shows the comparisons of OHAM-LS at $\alpha=1,0.9$, 0.8 , and 0.7 with RK4 at $\alpha=1$, which verifies the performance of the present method as an excellent tool for NLPCOPs. For $\alpha=1$, it can be seen that the approximate analytical solution agrees with the ideal solution. Thus, as $\alpha$ approaches 1 , the classical solution for the system is recovered. 


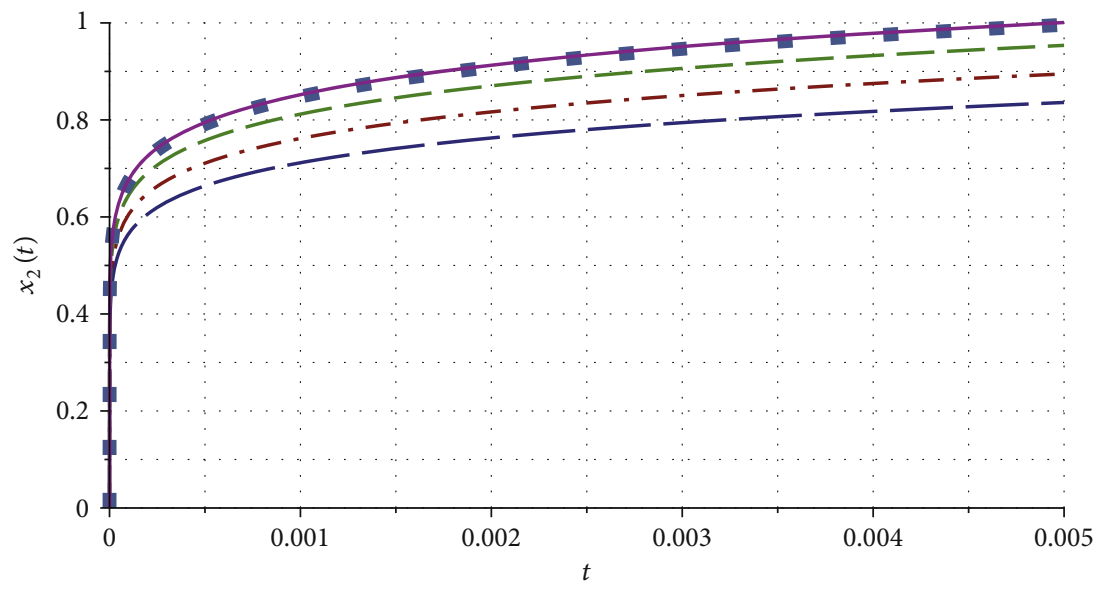

(a)

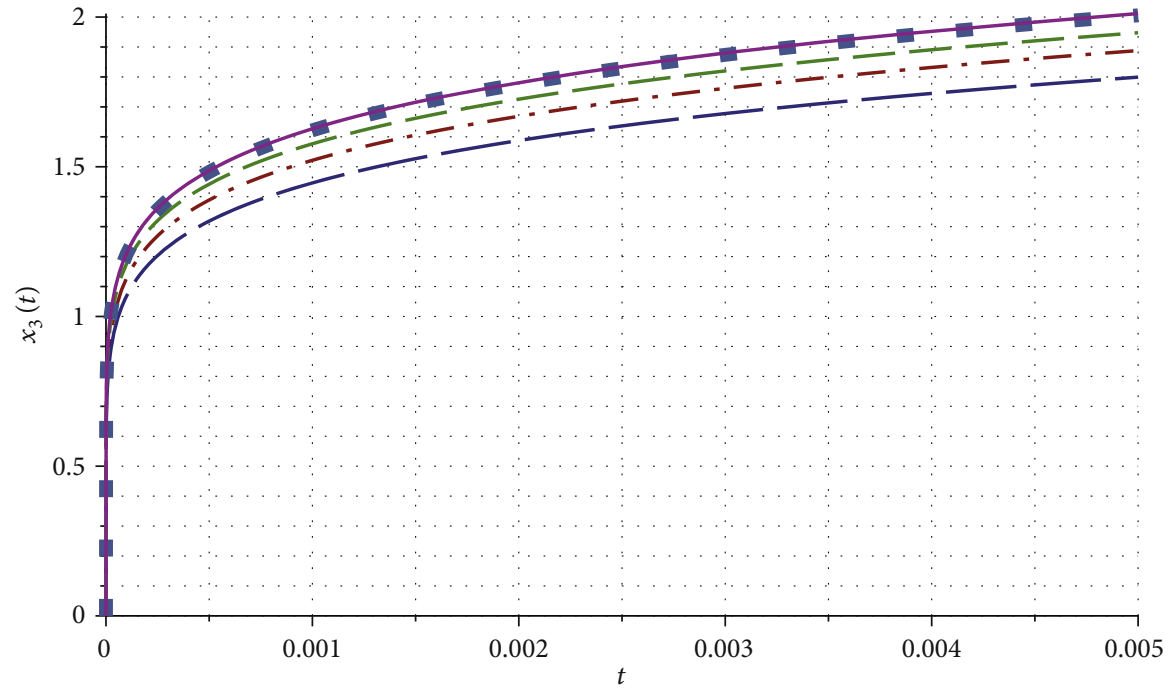

(b)

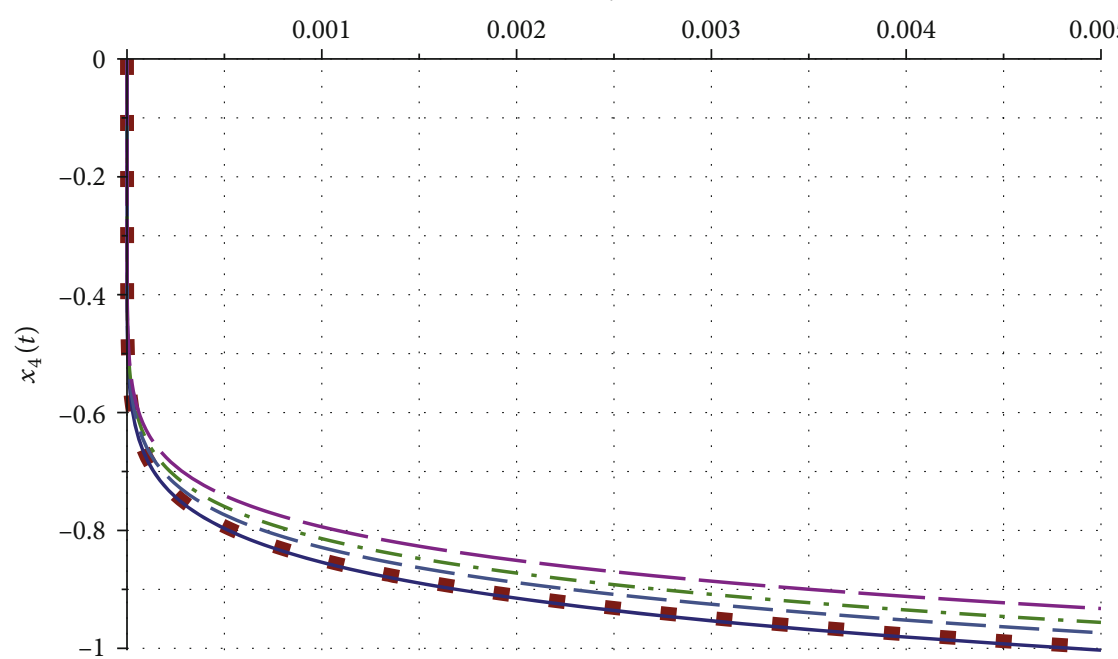

(c)

Figure 3: (a) Different values of $\alpha$ (OHAM-LS; $\alpha=1$ dot, $\alpha=0.9$ dash, $\alpha=0.8$ dash dot, and $\alpha=0.7$ long dash) and Rk4 ( $\alpha=1$, solid) at $x_{2}$. (b) Different values of $\alpha$ (OHAM-LS; $\alpha=1$ dot, $\alpha=0.9$ dash, $\alpha=0.8$ dash dot, and $\alpha=0.7$ long dash), and RK4 ( $\alpha=1$, solid) at $x_{3}$. (c) Different values of $\alpha$ (OHAM-LS; $\alpha=1$ dot, $\alpha=0.9$ dash, $\alpha=0.8$ dash dot, and $\alpha=0.7$ long dash), and RK4 ( $\alpha=1$, solid) at $x_{4}$. 


\section{Conclusions}

In this paper, we implemented OHAM-LS for solving nonlinear FOGBDS from the optimization problem. The fractional derivative is considered in a new conformable fractional derivative sense. The optimization minimization approach of the least square method helps to obtain optimal values of the $C^{s} j$ for accurate approximate analytical solutions. The comparisons between the fourth-order RungeKutta $(\alpha=1)$ and OHAM-LS show that our present method performs rapid convergence to the expected optimal solutions of the optimization problem. The results obtained are in close agreement with the exact solution, and those from the RK4 and OHAM-LS are reliable, dependable, and efficient for finding an approximate analytical solution for nonlinear FOGBDS optimization problem.

\section{Data Availability}

No data were used to support this study.

\section{Conflicts of Interest}

The authors declare that they have no conflict of interest.

\section{Authors' Contributions}

All authors have equal contributions and they read and approved the final version of the paper.

\section{References}

[1] Y. Ren, F. Guo, and Y. Li, "Nonlinear lagrangians for nonlinear programming based on modified fischer-burmeister NCP Function," Journal of Computational Mathematics, vol. 33, no. 4, pp. 396-414, 2015.

[2] F. Evirgen, "Solution of a class of optimization problems based on hyperbolic penalty dynamic framework," Acta Physica Polonica A, vol. 132, no. 3-II, pp. 1062-1065, 2017.

[3] Z. Meng, Q. Hu, C. Dang, and X. Yang, "An objective penalty function method for nonlinear programming," Applied Mathematics Letters, vol. 17, no. 6, pp. 683-689, 2004.

[4] S. Lian, S. Meng, and Y. Wang, "An objective penalty functionbased method for inequality constrained minimization problem," Mathematical Problems in Engineering, vol. 2018, Article ID 7484256, 7 pages, 2018.

[5] B. T. Nguyen, Y. Bai, X. Yan, and T. Yang, "Perturbed smoothing approach to the lower order exact penalty functions for nonlinear inequality constrained optimization," Tamkang Journal of Mathematics, vol. 50, no. 1, pp. 37-60, 2019.

[6] F. Scott, R. Conejeros, and V. S. Vassiliadis, "Constrained NLP via gradient flow penalty continuation: Towards self-tuning robust penalty schemes," Computers \& Chemical Engineering, vol. 101, pp. 243-258, 2017.

[7] D. G. Luenberger, "Convergence rate of a penalty-function scheme," Journal of Optimization Theory and Applications, vol. 7, no. 1, pp. 39-51, 1971.

[8] A. Beck and M. Teboulle, "Fast gradient-based algorithms for constrained total variation image denoising and deblurring problems," IEEE Transactions on Image Processing, vol. 18, no. 11, pp. 2419-2434, 2009.
[9] Y. Sun, Y. Liu, G. Wang, and H. Zhang, "Deep learning for plant identification in natural environment," Computational Intelligence and Neuroscience, vol. 2017, Article ID 7361042, 6 pages, 2017.

[10] H. Li, S. Liu, Y. C. Soh, and L. Xie, "Event-triggered communication and data rate constraint for distributed optimization of multiagent systems," IEEE Transactions on Systems, Man, and Cybernetics: Systems, vol. 48, no. 11, pp. 1908-1919, 2018.

[11] J. Zeng and W. Yin, "On non-convex decentralized gradient descent," IEEE Transactions on Signal Processing, vol. 66, no. 11, pp. 2834-2848, 2018.

[12] Y.-F. Pu, J.-L. Zhou, and X. Yuan, "Fractional differential mask: a fractional differential-based approach for multi-scale texture enhancement," IEEE Transactions on Image Processing, vol. 19, no. 2, pp. 491-511, 2010.

[13] P. Liu, Z. Zeng, and J. Wang, "Multiple Mittag-Leffler Stability of Fractional-Order Recurrent Neural Networks," IEEE Transactions on Systems, Man, and Cybernetics: Systems, vol. 47, no. 8, pp. 2279-2288, 2017.

[14] W. Yin, Y. Wei, T. Liu, and Y. Wang, "A novel orthogonalized fractional order filtered-x normalized least mean squares algorithm for feedforward vibration rejection," Mechanical Systems and Signal Processing, vol. 119, pp. 138-154, 2019.

[15] K. J. Arrow and L. Hurwicz, "On the stability of the competitive Equilibrium, I," Econometrica: Journal of the Econometric Society, vol. 26, no. 4, pp. 522-552, 1958.

[16] C. A. Botsaris, "Differential gradient methods," Journal of Mathematical Analysis and Applications, vol. 63, no. 1, pp. 177-198, 1978.

[17] H. Yamashita, "A differential equation approach to nonlinear programming," Mathematical Programming, vol. 18, no. 1, pp. $155-168,1980$.

[18] A. A. Brown and M. C. Bartholomew-Biggs, "Ode versus sqp methods for constrained optimization," Journal of Optimization Theory and Applications, vol. 62, no. 3, pp. 371-386, 1989.

[19] G. Adomian, "Solution of physical problems by decomposition," Computers Mathematics with Applications, vol. 27, no. 9-10, pp. 145-154, 1994.

[20] M. Baghani, M. Fattahi, and A. Amjadian, "Application of the variational iteration method for nonlinear free vibration of conservative oscillators," Scientia Iranica, vol. 19, no. 3, pp. 513-518, 2012.

[21] A. Yildirim and Y. Gülkanat, "Analytical approach to fractional zakharov-kuznetsov equations by he's homotopy perturbation method," Communications in Theoretical Physics, vol. 53, no. 6, pp. 1005-1010, 2010.

[22] S. Yang and A. Xiao, "An efficient numerical method for fractional differential equations with two Caputo derivatives," Journal of Computational Mathematics, vol. 34, no. 2, pp. 113-134, 2016.

[23] N. Özdemir and M. Yavuz, "Numerical solution of fractional black-scholes equation by using the multivariate padé approximation," Acta Physica Polonica A, vol. 132, no. 3-II, pp. 10501053, 2017.

[24] F. Evirgen and N. Özdemir, "Multistage adomian decomposition method for solving nlp problems over a nonlinear fractional dynamical system," Journal of Computational and Nonlinear Dynamics, vol. 6, no. 2, 2011.

[25] F. Evirgen and N. Özdemir, "A fractional order dynamical trajectory approach for optimization problem with HPM," Fractional Dynamics and Control, pp. 145-155, 2012. 
[26] S. Abbasbandy and M. Jalili, "Determination of optimal convergence-control parameter value in homotopy analysis method," Numerical Algorithms, vol. 64, no. 4, pp. 593-605, 2013.

[27] Y. F. Pu, J. L. Zhou, Y. Zhang, N. Zhang, G. Huang, and P. Siarry, "Fractional extreme value adaptive training method: fractional steepest descent approach," IEEE Transactions on Neural Networks and Learning Systems, vol. 26, no. 4, pp. 653-662, 2015.

[28] F. Evirgen, "Analyze the optimal solutions of optimization problems by means of fractional gradient based system using vim," An International Journal of Optimization and Control: Theories \& Applications (IJOCTA), vol. 6, no. 2, pp. 75-83, 2016.

[29] J. Wang, Y. Wen, Y. Gou, Z. Ye, and H. Chen, "Fractionalorder gradient descent learning of bp neural networks with Caputo derivative," Neural Networks, vol. 89, pp. 19-30, 2017.

[30] Y. Chen, Q. Gao, Y. Wei, and Y. Wang, "Study on fractional order gradient methods," Applied Mathematics and Computation, vol. 314, pp. 310-321, 2017.

[31] F. Evirgen, "Conformable fractional gradient based dynamic system for constrained optimization problem," Acta Physica Polonica A, vol. 132, no. 3-II, pp. 1066-1069, 2017.

[32] V. Marinca and N. Herisanu, "Application of optimal homotopy asymptotic method for solving nonlinear equations arising in heat transfer," International Communications in Heat and Mass Transfer, vol. 35, no. 6, pp. 710-715, 2008.

[33] M. Azimi, A. Mozaffari, and F. Ommi, "Application of galerkin optimal homotopy asymptotic method to shock wave equation," Journal of Advanced Physics, vol. 3, no. 1, pp. 35-38, 2014.

[34] R. Khalil, M. Al Horani, A. Yousef, and M. Sababheh, "A new definition of fractional derivative," Journal of Computational and Applied Mathematics, vol. 264, pp. 65-70, 2014.

[35] T. Abdeljawad, "On conformable fractional calculus," Journal of Computational and Applied Mathematics, vol. 279, pp. 5766, 2015.

[36] V. Marinca, N. Herisanu, C. Bota, and B. Marinca, "An optimal homotopy asymptotic method applied to the steady flow of a fourth-grade fluid past a porous plate," Applied Mathematics Letters, vol. 22, no. 2, pp. 245-251, 2009.

[37] F. Ghani, S. Islam, C. Ozel, L. Ali, M. M. Rashidi, and T. Hajjari, "Application of modified optimal homotopy perturbation method to higher order boundary value problems in a finite domain," Hacettepe Journal of Mathematics and Statistics, vol. 45, no. 4, pp. 1049-1060, 2016.

[38] N. Herisanu, V. Marinca, G. Madescu, and F. Dragan, "Dynamic response of a permanent magnet synchronous generator to a wind gust," Energies, vol. 12, no. 5, p. 915, 2019.

[39] V. Marinca and N. Herişanu, "On the flow of a Walters-type B' viscoelastic fluid in a vertical channel with porous wall," International Journal of Heat and Mass Transfer, vol. 79, pp. 146$165,2014$.

[40] N. Herisanu and V. Marinca, "Optimal homotopy asymptotic method to large post-buckling deformation of MEMS," MATEC Web of Conferences, vol. 148, article 13003, 2018.

[41] M. Sajid and T. Hayat, "Comparison of HAM and HPM methods in nonlinear heat conduction and convection equations," Nonlinear Analysis: Real World Applications, vol. 9, no. 5, pp. 2296-2301, 2008.
[42] E. Babolian, J. Saeidian, and M. Paripour, "Application of the Homotopy analysis method for solving equal-width wave and modified equal-width wave equations," Zeitschrift für Naturforschung $A$, vol. 64, no. 11, pp. 685-690, 2009.

[43] A. K. Alomari, M. S. M. Noorani, and R. Nazar, "Comparison between the homotopy analysis method and homotopy perturbation method to solve coupled Schrodinger-KdV equation," Journal of Applied Mathematics and Computing, vol. 31, no. 1-2, pp. 1-12, 2009.

[44] M. Kurulay, "Approximate analytic solutions of the modified Kawahara equation with homotopy analysis method," Advances in Difference Equations, vol. 2012, no. 1, 2012.

[45] C. A. Botsaris and D. H. Jacobson, "A newton-type curvilinear search method for optimization," Journal of Mathematical Analysis and Applications, vol. 54, no. 1, pp. 217-229, 1976.

[46] J. Liu and C. Ma, "A new nonmonotone trust region algorithm for solving unconstrained optimization problems," Journal of Computational Mathematics, vol. 32, no. 4, pp. 476-490, 2014.

[47] D. G. Luenberger and Y. Ye, Basic descent Methods, vol. 228 of International Series in Operations Research Management Science, Springer, 2016.

[48] X. Fang and Q. Ni, "A frame-based conjugate gradients direct search method with radial basis function interpolation model," Discrete Dynamics in Nature and Society, vol. 2017, Article ID 4082432, 9 pages, 2017.

[49] B. Baluch, Z. Salleh, and A. Alhawarat, "A new modified threeterm hestenes-stiefel conjugate gradient method with sufficient descent property and its global convergence," Journal of Optimization, vol. 2018, Article ID 5057096, 13 pages, 2018.

[50] C. Chen, Z. Wen, and Y. X. Yuan, "A general two-level subspace method for non-linear optimization," Journal of Computational Mathematics, vol. 36, no. 6, 2018.

[51] E. T. Hamed, H. I. Ahmed, and A. Y. Al-Bayati, "A new hybrid algorithm for convex nonlinear unconstrained optimization," Journal of Applied Mathematics, vol. 2019, Article ID 8728196, 6 pages, 2019.

[52] S. F. Mansouri and M. R. Maheri, "Constraint control method of optimization and its application to design of steel frames," Scientia Iranica, vol. 26, no. 4, pp. 2241-2257, 2019.

[53] Z. Lužanin, I. Stojkovska, and M. Kresoja, "Descent direction stochastic approximation algorithm with adaptive step sizes," Journal of Computational Mathematics, vol. 37, no. 1, pp. 76-94, 2019.

[54] K. Schittkowski, More test examples for nonlinear programming codes Springer-Verlag, vol. 282, Springer Science \& Business Media, 2012. 